\title{
Inclusão Digital: Mapeamento de Publicações sobre o Tema, na Área de Administração
}

\section{Digital Inclusion: Mapping of Publications on the Subject, in the Administration Field}

\author{
Mauri Leodir Löbler \\ Universidade Federal de Santa Maria - mllobler@ gmail.com \\ Larissa Medianeira Bolzan \\ Universidade Federal de Santa Maria - larissambolzan@ gmail.com \\ Flávio Issao Kubota \\ Universidade Federal de Santa Maria - flavioissao.kubota@ gmail.com \\ Silvia Amélia Mendonça Flores \\ Universidade Federal de Santa Maria - sisimflores@yahoo.com.br \\ Taciane Graciele Fanck Kich \\ Universidade Federal de Santa Maria - tacikich@ yahoo.com.br
}

\section{Resumo}

Tendo em vista a importância do estudo de inclusão digital para a administração, este artigo investigou como está sendo explorado o processo de inclusão digital em pesquisas e quais foram os principais resultados desses estudos. Para tanto, foram selecionados e analisados, 75 publicações científicas, nacionais e internacionais. Verificou-se que o processo de inclusão digital está sendo estudado de forma ampla e generalista, uma vez que é estudado o processo proposto por políticas públicas ou a interação com conteúdos na educação básica. Nenhum estudo investigou o processo de apropriação individual de conhecimentos sobre as ferramentas digitais e nem uma forma de mensurar o nível de autonomia sobre as TICs.

Palavras-Chave: Inclusão Digital, Divisão Digital, Excluído Digital.

\begin{abstract}
Considering the importance of the study of digital inclusion for administration, this study investigated how digital inclusion process is being explored in researches and what were the main results of these researches. Hence, we selected and analyzed 75 scientific publications, national and international. We verified that the digital inclusion process is studied in a wide and generalist manner, since it is studied the proposed process by public policies or content interaction in basic education. No study has investigated the process of individual appropriation of knowledge about digital tools, and there was not proposed a way to measure the level of digital inclusion of autonomy.
\end{abstract}

Keywords: Digital Inclusion, Digital Divide, Digital Exclusion.

\section{Introdução}

As novas tecnologias, para alguns, trouxeram grandes esperanças, a promessa do desenvolvimento e de um futuro melhor, enquanto para outros, as ferramentas digitais aumentaram o abismo entre as nações e dentro das nações (Carvalho, 2009). Segundo entendimento de Cubillos e Silva (2009) isso ocorre devido ao alto preço que o mercado cobra pelas Tecnologias de Informação e Comunicação (TICs), limitando o acesso de 
grande parte da população que não pode pagar pelo mesmo, constituindo, assim, uma massa de excluídos. A esse respeito, Teixeira (2001) afirma que foi criada uma nova modalidade de exclusão social, configurada - sob uma visão simplista - a partir de uma divisão entre aqueles que possuem, ou não, acesso às TICs.

Batista (2006) e Alonso et al. (2010) consideram difícil encontrar alternativas que visem à inclusão digital em um país com um alto índice de analfabetismo e elevada concentração da renda e riqueza, ou seja, problemas que precisam ser sanados com urgência. Mesmo assim, os autores afirmam que não há como pensar em inclusão digital em segundo plano, visto que o desenvolvimento das tecnologias se dá cada vez mais rapidamente e o abismo existente entre incluídos e excluídos tende a aumentar.

Sob o ponto de vista da Administração, estudar o processo de inclusão digital torna-se relevante ao passo que os equipamentos utilizados em processos produtivos estão cada vez mais automatizados, a exemplo disso, os tornos programados por Comando Numérico Computacional $(\mathrm{CNC})$ e as prensas hidráulicas acionadas por comandos digitais. Segundo Fonseca e Lima (2007) a introdução de novas tecnologias gerou uma ruptura nos saberes dos trabalhadores, criando-se uma lacuna entre o conhecimento técnico operacional (necessário anterior à introdução de novas tecnologias) e o conhecimento atualmente exigido (conhecimento técnico com o uso de novas tecnologias). Nesse sentido, conforme os mesmos autores, a acrescente adoção de tecnologia na indústria e, portanto o aumento do gap de conhecimento é responsável por grande número de acidentes de trabalho. Dessa forma, emerge o objetivo deste trabalho: investigar como está sendo explorado o processo de inclusão digital e quais foram os principais resultados dos estudos a respeito do tema.

Este trabalho está estruturado em mais cinco seções, além da introdução. $\mathrm{Na}$ seção dois, aborda-se o referencial teórico que balizou a realização deste estudo. $\mathrm{Na}$ seção três, é apresentada a arquitetura metodológica que possibilitou a operacionalização da pesquisa. Na seção quatro são apresentados os resultados alinhados ao objetivo proposto. Por fim, na seção cinco, as considerações finais. $\mathrm{Na}$ sequência, as referências bibliográficas utilizadas no estudo em questão.

\section{Inclusão Digital}

Explorando o panorama nacional de inclusão digital, percebe-se que as TICs já foram inseridas na sociedade. Nos estudos de Batista (2006) e Young (2006) a parcela menos favorecida da população e os idosos foram considerados inicialmente como excluídos digitais. Em seguida, os autores expuseram, aos mesmos, o acesso obrigatório a caixas eletrônicos, devido às políticas sociais como o Bolsa-Familia e os benefícios do INSS, e também a urna eletrônica. Quanto a celulares, pagers, palms, notebooks e o crescente uso da internet como meio de comunicação e aprendizado (Fernandes e Ross, 2006; Spinello e Teixeira, 2008), compartilha-se do entendimento de Fernandes e Ross (2006) e Moraes et al. (2009), que afirmam que esse cenário surgiu a partir de sinais da expansão e imposição de ditames do mercado digital.

Quanto ao processo de inclusão digital, Medeiros Neto e Miranda (2010) afirmam que a democratização de acesso e de conectividade é apenas o primeiro passo. Alonso et al. (2010) acrescentam as etapas de alfabetização digital e posterior possibilidade de atualização. Essa possibilidade é explicada por Teixeira (2005), que ao considerar o processo de inclusão digital dinâmico e provisório, enfatiza o fato de as TICs serem reinventadas e aprimoradas com grande rapidez, exigindo constante desenvolvimento de habilidades necessárias à interação, com e através das novas tecnologias. 
Conforme o entendimento de Freire (2006), a expressão "inclusão digita"' tem um significado bastante complexo na conjuntura atual. Para Young (2006), inclusão digital significa capacitar indivíduos para uso efetivo de recursos tecnológicos, com o objetivo de desenvolvimento individual e coletivo, econômico e político. Pinho et al. (2008) utiliza-se da expressão "apropriação do conhecimento" sobre as tecnologias de informação e comunicação para definir inclusão digital. Apropriar-se das tecnologias significa desenvolver e aperfeiçoar habilidades que vão de tarefas básicas, como escrever uma mensagem ou reconhecer um spam, a atividades complexas, como pesquisar de maneira eficaz, acessar serviços ou produzir um vídeo digital e transmiti-lo via web (Pinho et al., 2008).

De acordo com Cusin e Vidotti (2009), inclusão digital trata de propiciar fluência tecnológica e utilização crítica das ferramentas digitais, com o objetivo de alavancar a aprendizagem significativa, autônoma e contínua, além de mobilizar o exercício da cidadania e oportunizar a produção de conhecimentos necessários à melhoria das condições de vida das pessoas e da sociedade. Martins e Lucas (2009) trouxeram uma concepção de inclusão digital que tratou da capacidade de utilização de equipamentos eletrônicos a fim de acessar e manejar informações em ambiente eletrônico. Essa concepção foca no desenvolvimento de habilidades e conhecimentos puramente mecânicos, basicamente voltados ao entendimento do funcionamento de hardware, software e aplicativos. Para Ferro et al. (2011) a inclusão digital deve proporcionar a habilidade para usar as TICs de forma eficaz, ou seja, tem como responsabilidade promover desenvolvimento das competências técnicas (habilidades necessárias para operar hardware e software) e educacionais (habilidades necessárias para a compreensão da informação).

Nesse sentido, considerando a literatura sobre o tema, chega-se a conclusão de que o processo de inclusão digital é conceituado como: o processo dinâmico que, através do acesso as TICs, torna possível a apropriação do conhecimento sobre tecnologia de informação e comunicação, para que o indivíduo possa desenvolver e aperfeiçoar habilidades capazes de propiciar autonomia sobre as ferramentas digitais e utilização crítica das mesmas. É imprescindível, também, que o processo de inclusão digital possibilite o aprimoramento da interação, ou seja, atualização do conhecimento.

\section{Método}

Para a realização deste estudo, primeiramente, buscou-se definir as bases em que seriam pesquisados estudos sobre inclusão digital. $\mathrm{O}$ levantamento se deu em anais de eventos nacionais relevantes para a área de Administração, e na sequência, periódicos nacionais e internacionais cujo tema central é Tecnologia de Informação e Desenvolvimento Social e Sociedade no período compreendido entre os anos de 2006 até agosto/2011, o que denota cinco anos de análise. Quanto aos eventos, foram pesquisados aqueles promovidos pela ANPAD (Associação Nacional dos Programas de Pós-Graduação em Administração), visto ser o órgão máximo da Pós-Graduação em Administração no Brasil. No que se refere aos periódicos, foram pesquisados todos aqueles constantes na relação de estratificação de qualidade da Coordenação de Aperfeiçoamento de Pessoal de Nível Superior (Qualis/Capes), da área de Administração, disponível no sítio www.qualis.capes.gov.br. Ainda, com relação aos periódicos, foi utilizada a metabusca da base de dados dos Periódicos Capes, disponível no sítio www.periodicos.capes.gov.br. Em cada uma das bases pesquisadas foi verificado a existência de uma das seguintes expressões-chaves no título do artigo: Inclusão Digital, Digital Inclusion, Divisão Digital, Digital Divide, Incluído ou/e Excluído Digital e 
Digital Exclusion. Quando uma das referidas expressões constava no título, o artigo era selecionado e fazia parte da análise proposta.

No que tange à classificação do método dos artigos selecionados, foi realizada a categorização segundo o modelo utilizado por Löbler et al. (2011), que realizaram um mapeamento metodológico de artigos, considerando o caráter e a natureza da pesquisa, a forma como foram levantados os dados e a unidade de análise. Quanto aos resultados, esses também foram analisados, porém não classificados. Os autores deste estudo acreditam que a contribuição maior seria uma compilação de todas as descobertas. Então, nesse trabalho foi realizada essa compilação e apresentada em forma de tabela (Tabela 2), onde também foi citado cada autor.

\section{Resultados}

Quanto aos artigos selecionados de eventos, foram encontrados trabalhos com a temática nos seguintes: Encontro Nacional da ANPAD - ENANPAD (2), Encontro Nacional da Administração de Informação - ENADI (1), Encontro de Administração Pública e Governança - ENAPG (2) e Simpósio de Gestão da Inovação Tecnológica (3). No que se refere aos periódicos, foram selecionados artigos dos seguintes: Ciência \& Saúde Coletiva (1), Ciência da Informação (3), Inclusão Social (11), Liinc em Revista (7), Perspectiva em Ciência da Informação (1), Revista Brasileira de Educação (1), Revista Espaço Acadêmico (1), Revista Novas Tecnologias na Educação - RENOTE (14), Archives of Gerontology and Geriatrics (1), Barbarói (1), British Journal of Social Work (2), Communication \& Ethics in Society (1), Computers \& Education (1), European Sociological Review (1), Geoforum (1), Government Information Quarterly (3), Human Relations (1), Information Economics and Policy (1), Information Systems Research (1), Information Technology for Development (1), International Journal of Educational Development (1), Journal of Travel Research (1), Journal of Information, Media, Culture \& Society (1), New Media \& Society (1), Poetics (2), Revista de Economía Política de las Tecnologías de la Información y Comunicación (1), Telecommunications Policy (2), Telematics and Informatics (2), The Computer Journal (1), World Development (1).

Foram selecionados 75 artigos, sendo que 48 desses possuíam no título a expressão "Inclusão Digital", 6 "Digital Inclusion", 19 "Digital Divide", 1 "Incluído/Excluído Digital" e 1 "Digital Exclusion". Quanto à relação entre os estudos nacionais e internacionais (apresentados na Figura 01), pode-se observar que, no período de 2006 a 2010, a produção nacional sobre o tema inclusão digital manteve-se superior a internacional e no ano de 2011 apresentou uma queda.

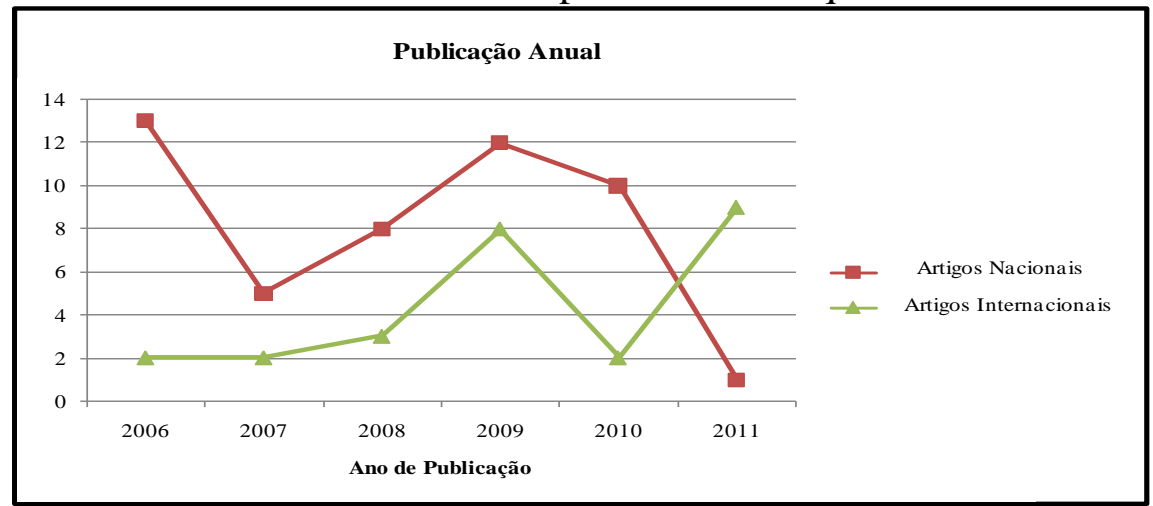

Figura 01 - Publicação anual de artigos 
Em relação à classificação dos periódicos em que os artigos foram publicados, os dados são apresentados na Figura 02, que objetiva mostrar a relação entre os artigos nacionais e internacionais, conforme a estratificação de qualidade da CAPES, referente ao triênio 2007-2009. Destaca-se que, dos estudos analisados, apenas oito foram publicados em eventos - portanto, não fazem parte da estratificação apresentada na figura. Para melhor compreensão, salienta-se que a série 01 (representada pela cor verde) engloba os artigos nacionais e a série 02 (cor laranja) os artigos internacionais.

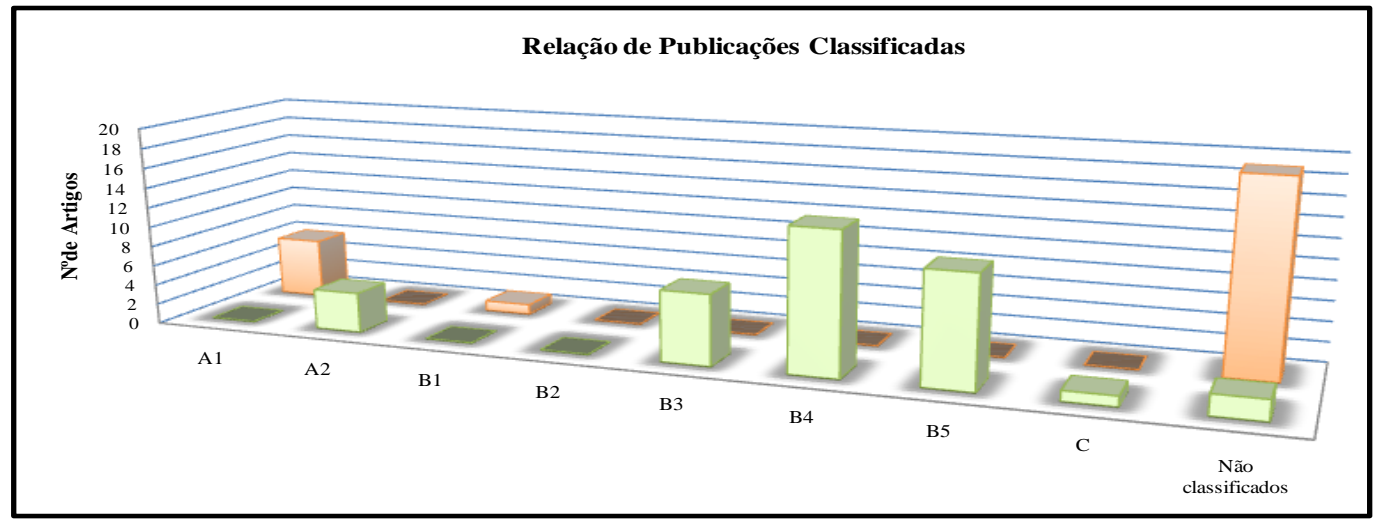

Figura 02 - Relação entre publicações nacionais e internacionais

4.1. Como está sendo estudado o tema Inclusão Digital?

$\mathrm{Na}$ primeira etapa da análise de resultados desse estudo foi verificado se a pesquisa tratava-se de um estudo empírico ou teórico, observou-se que do total analisando, 59 estudos são empíricos e 16 teóricos. Dos empíricos, 41 são nacionais e 18 internacionais. Dos 16 teóricos, 8 são nacionais e 8 internacionais. Quanto aos estudos teóricos ainda cabe destacar que apenas, dos artigos internacionais, 1 é bibliométrico, o restante são bibliográficos, ou seja, o assunto foi exposto sem uma sistemática para sua captura (Gil, 2010). A Figura 03 apresenta a análise de estudos empíricos, conforme o procedimento metodológico utilizado por Löbler et al. (2011).

\begin{tabular}{|c|c|c|c|c|c|c|c|c|}
\hline \multicolumn{9}{|c|}{ ARTIGOS NACIONAIS } \\
\hline \multicolumn{2}{|c|}{$\begin{array}{c}\text { Caráter da } \\
\text { Pesquisa }\end{array}$} & \multicolumn{2}{|c|}{ Natureza da Pesquisa } & \multicolumn{2}{|c|}{ Tipo de Pesquisa } & \multicolumn{2}{|c|}{$\begin{array}{c}\text { Levantamento de } \\
\text { Dados }\end{array}$} & \multirow[t]{2}{*}{$\begin{array}{c}\text { Unidade de } \\
\text { Análise }\end{array}$} \\
\hline & $\begin{array}{l}\mathrm{n}^{0} \text { de } \\
\text { pesq. }\end{array}$ & & $\begin{array}{l}\mathrm{n}^{\mathbf{0}} \text { de } \\
\text { pesq. }\end{array}$ & & $\begin{array}{l}\mathrm{n}^{0} \text { de } \\
\text { pesq. }\end{array}$ & & $\begin{array}{l}\mathrm{n}^{\mathbf{0}} \text { de } \\
\text { pesq. }\end{array}$ & \\
\hline \multirow{6}{*}{ 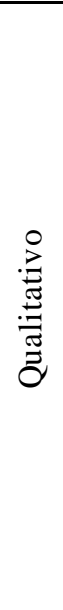 } & \multirow{6}{*}{30} & \multirow{6}{*}{ Descritiva } & \multirow{6}{*}{11} & Documental & 1 & $\begin{array}{l}\text { Dados } \\
\text { Secundários }\end{array}$ & 1 & \begin{tabular}{|l|} 
Portal de \\
Inclusão \\
Digital \\
\end{tabular} \\
\hline & & & & \multirow{5}{*}{$\begin{array}{l}\text { Estudo de } \\
\text { Caso }\end{array}$} & \multirow{5}{*}{7} & $\begin{array}{l}\text { Entrevistas } \\
\text { semi- } \\
\text { estruturadas }\end{array}$ & 1 & $\begin{array}{l}\text { Projeto de } \\
\text { Inclusão }\end{array}$ \\
\hline & & & & & & \multirow{4}{*}{$\begin{array}{l}\text { Observação } \\
\text { não- } \\
\text { participante }\end{array}$} & \multirow{4}{*}{6} & \begin{tabular}{|l|} 
Biblioteca \\
Nacional \\
\end{tabular} \\
\hline & & & & & & & & $\begin{array}{l}\text { Comunidade de } \\
\text { Agricultura } \\
\text { Familiar }\end{array}$ \\
\hline & & & & & & & & $\begin{array}{l}\text { Curso EAD } \\
\text { para } \\
\text { Professores }\end{array}$ \\
\hline & & & & & & & & Escola \\
\hline
\end{tabular}




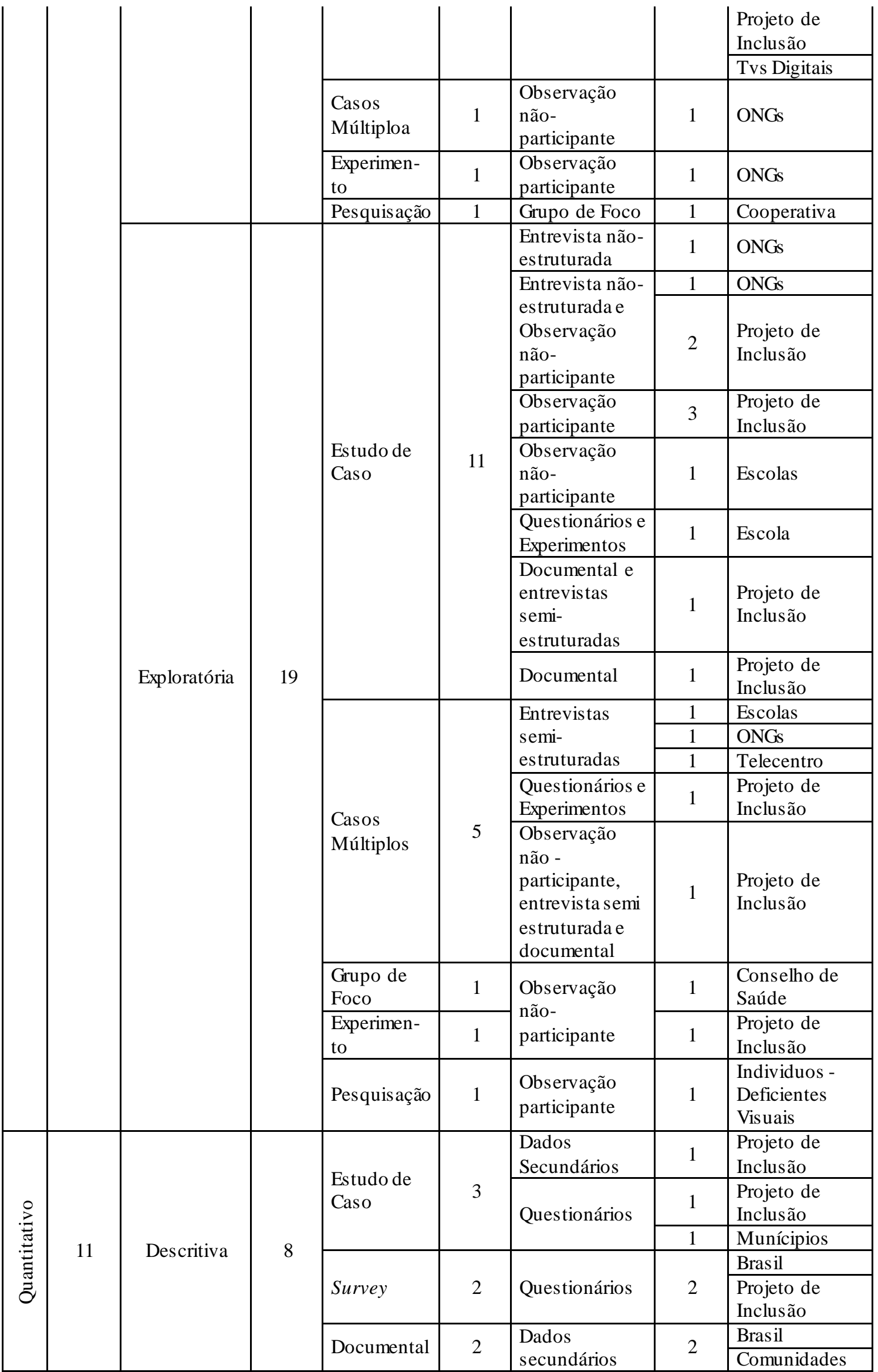




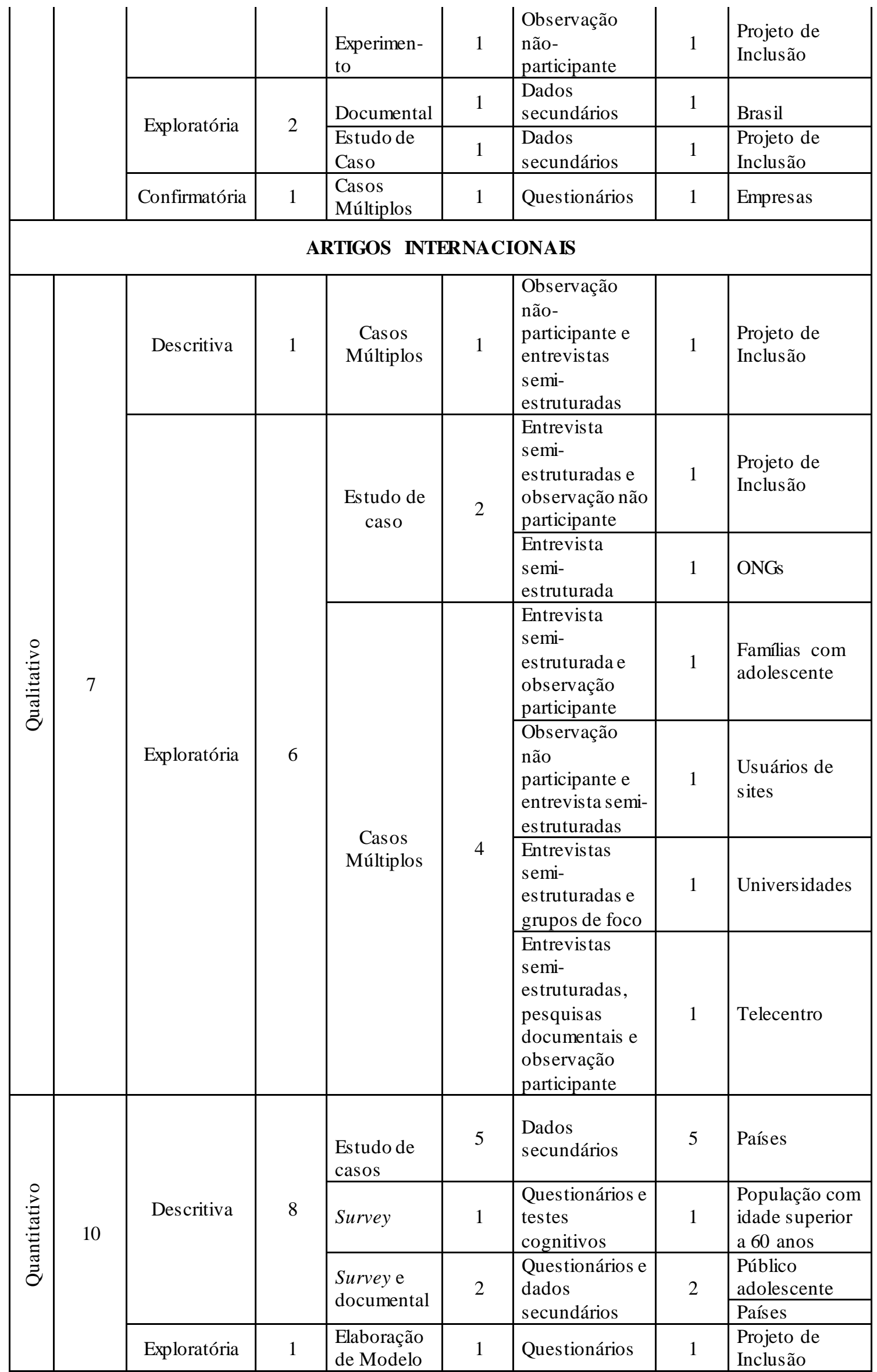




\begin{tabular}{|c|c|c|c|c|c|c|c|c|}
\hline & & Confirmatória & 1 & Survey & 1 & Questionários & 1 & Escolas \\
\hline 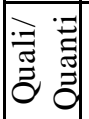 & 1 & $\begin{array}{c}\text { Fase } \\
\text { exploratória e } \\
\text { descritiva }\end{array}$ & 1 & Survey & 1 & $\begin{array}{l}\text { Questionários e } \\
\text { entrevistas em } \\
\text { profundidade }\end{array}$ & 1 & Escolas Rurais \\
\hline
\end{tabular}

Figura 03 - Análise do método dos artigos pesquisados

\subsection{Principais Resultados dos Estudos em Inclusão Digital}

Quanto aos principais resultados, esses se encontram compilados na Figura 04.

\begin{tabular}{|c|c|}
\hline $\begin{array}{l}\text { RESULTADO/CONTRIB UIÇÃO } \\
\text { DA PESQUISA }\end{array}$ & AUTOR \\
\hline $\begin{array}{l}\text { Maior nível de educação e mais } \\
\text { idade são fatores facilitadores para } \\
\text { Inclusão Digital. }\end{array}$ & Roland et al., 2011; Ordones et al., 2011. \\
\hline $\begin{array}{l}\text { As ONGs têm papel fundamental na } \\
\text { elaboração e execução de políticas } \\
\text { públicas de inclusão digital. }\end{array}$ & $\begin{array}{l}\text { Roland et al., 2011; Ganesh e Barber, 2011; Alonso et al., 2010; } \\
\text { Cubillos e Silva, 2009. }\end{array}$ \\
\hline $\begin{array}{l}\text { A inclusão digital favorece o } \\
\text { desenvolvimento da região. }\end{array}$ & Roland et al., 2011; Moraes et al., 2009. \\
\hline $\begin{array}{l}\text { Existe uma estreita relação entre IDH } \\
\text { e Inclusão digital. }\end{array}$ & $\begin{array}{l}\text { Ross et al., 2006; Moraes et al., 2009; Rabia et al., 2006; } \\
\text { Alonso et al., 2010; Minghetti e Buhalis, 2011. }\end{array}$ \\
\hline $\begin{array}{l}\text { A educação é beneficiada com os } \\
\text { programas de inclusão digital. }\end{array}$ & $\begin{array}{l}\text { Telles e Jóia, 2011; Telles e Jóia, 2010; Marcon, 2010; Mello e } \\
\text { Teixeira, 2008; Marcon e Teixeira, 2009; Buzato, 2008. }\end{array}$ \\
\hline $\begin{array}{l}\text { A inclusão digital apresenta relação } \\
\text { com a auto estima. }\end{array}$ & $\begin{array}{l}\text { Telles e Jóia, 2011; Telles e Jóia, 2010; Marcon, 2010; Griebler } \\
\text { et al., 2010; Barbosa et al., 2008; Marcon e Teixeira, 2009; } \\
\text { Silveira et al., 2007; Silveira et al., 2010; Wolf e Carrion, 2010; } \\
\text { Estabel et al., 2006; Ordones et al., 2011. }\end{array}$ \\
\hline $\begin{array}{l}\text { A inclusão digital impulsiona a } \\
\text { possibilidade de inclusão social. }\end{array}$ & $\begin{array}{l}\text { Griebler et al., 2010; Roland et al., 2011; Marcon e Teixeira, } \\
\text { 2009; Madon, 2009; Brandão e Tricólli, 2006; Moraes et al. } \\
\text { 2009; Moraes et al., 2009; Rabia et al., 2006. }\end{array}$ \\
\hline $\begin{array}{l}\text { As TICs tornam o ensino mais } \\
\text { agradável, do ponto de vista dos } \\
\text { estudantes. }\end{array}$ & $\begin{array}{l}\text { Telles e Jóia, 2011; Telles e Jóia, 2010; Marcon, 2010; Mello e } \\
\text { Teixeira, 2008; Marcon e Teixeira, 2009; Foresti e Teixeira, } \\
\text { 2006; Buzato, 2008; Medeiros Neto e Miranda, 2010; Martins e } \\
\text { Lucas, 2009; Ferro et al., 2011; Waycott et al., 2010; Salinas e } \\
\text { Sanchez, 2009. }\end{array}$ \\
\hline $\begin{array}{l}\text { Os professores têm um papel } \\
\text { estratégico na inclusão digital através } \\
\text { da educação. }\end{array}$ & $\begin{array}{l}\text { Marcon e Teixeira, 2009; Marcon, 2010; Santos e Giraffa, 2010; } \\
\text { Salinas e Sanchez, } 2009 .\end{array}$ \\
\hline $\begin{array}{l}\text { A educação é um pré-requisito para a } \\
\text { inclusão digital. }\end{array}$ & $\begin{array}{l}\text { Mattos e Chagas, 2008; Mairs, 2007; Akhras, 2010; Batista, } \\
\text { 2006; Ferro et al., 2011. }\end{array}$ \\
\hline $\begin{array}{l}\text { A inclusão digital impulsiona o } \\
\text { desenvolvimento. }\end{array}$ & $\begin{array}{l}\text { Rezende, 2006; Griebler et al., 2010; Marcon e Teixeira, 2009; } \\
\text { Madon, 2009; Tripp, 2011; Souza e Coelho, 2009; Pinho et al., } \\
\text { 2008; Fernandes e Ross, 2006; Castro, 2008; Young, 2006; } \\
\text { Fiho, 2007; Martins e Lucas, 2009; Ferro et al., 2011; Fuchs e } \\
\text { Horak, 2008. }\end{array}$ \\
\hline $\begin{array}{l}\text { O apoio do governo é importante } \\
\text { para o desenvolvimento de } \\
\text { programas de inclusão digital. }\end{array}$ & $\begin{array}{l}\text { Madon, 2009; Carvalho, 2009; Cubillos e Silva, 2009; Çilan et } \\
\text { al., 2009. }\end{array}$ \\
\hline $\begin{array}{l}\text { A variável classe social é um } \\
\text { indicador de inclusão digital. }\end{array}$ & $\begin{array}{l}\text { Moraes et al., 2009; Tripp, 2011; Mattos e Chagas, 2008; Ferro } \\
\text { et al., } 2011 .\end{array}$ \\
\hline $\begin{array}{l}\text { Há necessidade de avaliar os } \\
\text { programas de inclusão digital. }\end{array}$ & Buzato, 2008; Alonso et al., 2010; Brandão e Tricólli, 2006. \\
\hline $\begin{array}{l}\text { Grande parte da população rural são } \\
\text { excluídos digitais. }\end{array}$ & $\begin{array}{l}\text { Warren, 2007; Medeiros Neto e Miranda, 2010; Helbig et al., } \\
\text { 2009; Warren, 2007; Salinas e Sanchez, 2009; Vicente e Lopez, } \\
\text { 2011. }\end{array}$ \\
\hline $\begin{array}{l}\text { Idade, presença de algum tipo de } \\
\text { deficiências, baixo nível de renda e } \\
\text { pouca educação são barreiras para }\end{array}$ & $\begin{array}{l}\text { Wong et al., 2009; Cusin e Vidotti, 2009; Medeiros Neto e } \\
\text { Miranda, 2010; Helbig et al., } 2009 .\end{array}$ \\
\hline
\end{tabular}




\begin{tabular}{|l|l|}
\hline inclusão digital. & \\
\hline $\begin{array}{l}\text { A legislação pode favorecer a } \\
\text { inclusão digital. }\end{array}$ & $\begin{array}{l}\text { Wong et al., 2009; Rothberg, 2009; Cusin e Vidotti, 2009; } \\
\text { Medeiros Neto e Miranda, 2010. }\end{array}$ \\
\hline $\begin{array}{l}\text { O comportamento da comunidade } \\
\text { pode ser uma barreira para a inclusão } \\
\text { digital. }\end{array}$ & Henriksen et al., 2007; Medeiros Neto e Miranda, 2010. \\
\hline $\begin{array}{l}\text { Não há indicadores efetivos para } \\
\text { inclusão digital, exclusão digital ou } \\
\text { divisão digital. }\end{array}$ & Van Dijk, 2006; Gilbert, 2008; Mattos e Chagas, 2008. \\
\hline
\end{tabular}

Figura 04 - Síntese dos resultados

\section{Considerações Finais}

O tema inclusão digital, por ser bastante recente, abrangente e importante para a administração de empresas, justifica a realização deste trabalho, que teve como objetivo investigar como o referido tema está sendo estudado e quais os principais resultados obtidos através das pesquisas. Quanto à forma como o tema é explorado nos estudos analisados, foi observado que, tanto no âmbito nacional quanto internacional, há predominância de estudos empíricos, de natureza qualitativa, caráter exploratório, do tipo estudos de caso e quanto à coleta de dados, há uma maior utilização de dados secundários. Além disso, cabe salientar que como unidade de análise, foi observada massiva utilização dos programas de inclusão digital. Referente aos resultados, esses foram compilados e apresentados brevemente em forma de tabela (Tabela 02), onde também foi citado cada autor.

Pode-se considerar que o processo de inclusão digital está sendo estudado de forma ampla e generalista, uma vez que é estudado o processo de inclusão proposto por políticas públicas ou a interação com conteúdos de educação básica. Nenhum estudo investigou o processo de apropriação individual de conhecimentos sobre as ferramentas digitais, corroborando com as conclusões do estudo de Notten et al. (2008). Da mesma forma como não foi proposta uma maneira de mensurar o nível de inclusão digital de autonomia sobre as TICs (Van Dijk, 2006; Gilbert, 2008; Mattos e Chagas, 2008). Dessa forma, para pesquisas futuras, sugere-se que sejam analisados processos individuais de apropriação de conhecimentos digitais, assim como proposta de indicadores do processo inclusão digital. Como limitação desta pesquisa, pode-se citar o acesso a publicações disponíveis online.

\section{Referencias Bibliográficas}

ALONSO, L. B. N; FERNEDA, E.; SANTANA G. P. Inclusão Digital e Inclusão Social: Contribuições Teóricas e Metodológicas. Barbarói, Rio Grande do Sul, n.32, p.154-177, 2010.

BATISTA, S. G. A inclusão digital: programas governamentais e o profissional da informação - reflexões. Inclusão Social, Brasîlia, v.1, n.2, p.23-30, 2006.

CARVALHO, O. B. M. Os "incluídos digitais" são "incluídos sociais"? Estado, mercado e a inserção dos indivíduos na sociedade da informação. Liinc em Revista, Rio de Janeiro, v.5, n.1, p.19-31, 2009.

CUBILlOS, D. A. C. V.; SILVA, A. S. C. Inclusão Digital: Sistemas de Engrenagens. Liinc em Revista. Rio de Janeiro. v.5, n.1, p.32-44, 2009.

CUSIN, C. A.; VIDOTTI, S. A. B. G. Inclusão Digital via Acessibilidade Web. Liinc em Revista, Rio de Janeiro, v.5, n.1, p.45-65, 2009.

FERNANDES, J. M.; ROSS, S. D. Pequenas Ilhas de Inclusão em um Oceano de Exclusão Digital: Uma Análise da População Brasileira com Acesso à Internet ao longo 
do tempo, espaço e status socioeconômico. In: Encontro Nacional de administração Pública e Govemança, 2, 2006, Salvador. Anais... São Paulo: ANPAD, 2006.

FERRO, E.; HELBIG, N. C.; GARCIA, J. R. G. The role of IT literacy in defining digital divide policy needs. Government Information Quarterly. v.28, p.3-10, 2011.

FONSECA E. D.; LIMA, F. P. A. Novas tecnologias construtivas e acidentes na construção civil: o caso da introdução de novo sistema de escoramento de formas de laje. Revista Brasileira de Saúde Ocupacional, São Paulo, v.32, n.115. p.41-51. 2007. FREIRE, I. M. Janelas da Cultura Local: Abrindo Oportunidades para Inclusão Digital de Comunidades. Ciência da Informação, Brasîlia, v.35, n.3, p.227-235, set./dez. 2006.

GILBERT, M. R.; MASUCCI, M.; HOMKO, C.; BOVE, A. A. Theorizing the digital divide: Information and communication technology use frameworks among poor women using a telemedicine system. Geoforum, v.39, n.2, p.912-925, 2008.

LÖBLER, M. L.; VISENTINI, M. S.; FERREIRA, A. C. Transversalidade entre Cognição e Sistemas de Informação: Um Mapeamento dos Principais Periódicos Internacionais. Organização e Sociedade, Bahia, v.18, n.56, p.153-173, 2011.

MARTINS, T. S.; LUCAS, E. R. O. Os Programas de Inclusão Digital do Governo Federal sob a óptica da Competência Informacional. Liinc em Revista, Rio de Janeiro, v.5, n.1, p.82-99, 2009.

MATTOS, F. A. M.; CHAGAS, G. J. N. Desafios para a Inclusão Digital no Brasil. Perspectivas em Ciências da Informação, Bahia, v.13, n.1, p.67-95, 2008.

MEDEIROS NETO, B.; MIRANDA, A. L. C. Uso da Tecnologia e Acesso à Informação pelos Usuários do Urograma GESAC e de Ações de Inclusão Digital do Governo Brasileiro. Inclusão Social, Distrito Federal, v.3, n.2, p.81-96, jan/jun, 2010.

MORAES, I. H. S.; VEIGA, L. VASCONCELlOS. M. M.; SANTOS, S. R. F. R. Inclusão Digital e Conselheiros de Saúde: Uma Política para a Redução da Desigualdade Social no Brasil. Ciência \& Saúde Coletiva, Rio de Janeiro, v.14, n.3, p.879-888, mai./jun. 2009.

NOTTEN, N.; PETER, J.; KRAAYKAMP, G.; VALKENBURG, P. M. Research Note: Digital Divide Across Borders - A Cross-National Study of Adolescents' Use of Digital Technologies. European Sociological Review, v.25, n.5, p.551-560, 2008.

PINHO, F. R.; OLIVEIRA, D. M.; XIMENES, P. K. M.; ROCHA, M. C. L.; ANDRADE, R. J. C. Empreendedorismo Social com Inclusão Digital: O Caso Pirambu Digital. In: XXV Simpósio de Gestão da Inovação Tecnológica, 25, 2008, Brasilia, Anais: XXV Simpósio de Gestão da Inovação Tecnológica.

SPINELLO, S.; TEIXEIRA, A. C. Medindo a interatividade em um ambiente de autoria hipermídia: qualificando processos de inclusão digital. Revista de Novas Tecnologias na Educação, Rio Grande do Sul, v.6, n.1, 2008.

TEIXEIRA, A. C. Internet e Democratização do Conhecimento: Repensando o Processo de Exclusão Social, no estado do Rio grande do Sul. Passo Fundo: UPF, 2001. 132p. Dissertação de Mestrado.

TEIXEIRA, A. C. Formação Docente e Inclusão Digital: A Análise do Processo de Emersão Tecnológica de Professores, no estado do Rio grande do Sul. Porto Alegre: UFRGS, 2005. 126p. Tese de Doutorado.

YOUNG, R. A Inclusão Digital e as Metas do Milênio. Inclusão Social, Distrito Federal, v.1, n.2, p.96-99, abr./set. 2006.

VAN DIJK, J. A. G. M. Digital divide research, achievements and shortcomings. Poetics, v.34, n.4-5, p.221-235, 2006. 
As referências pertencentes à Tabela 2, Síntese dos Resultados, não foram inseridas nas Referencias Bibliográficas devido ao limite de laudas, porém os autores disponibilizarão mediante contato. 